Pure and Applied Mathematics Quarterly

Volume 6, Number 3

(Special Issue: In honor of

Joseph J. Kohn, Part 1 of 2)

$843-874,2010$

\title{
The Oka Principle for Sections of Stratified Fiber Bundles
}

\author{
Franc Forstnerič \\ Dedicated to Professor J.J.Kohn on the occasion of his 75th birthday
}

\begin{abstract}
Let $Y$ be a complex manifold with the property that every holomorphic map from a neighborhood of a compact convex set $K$ in a complex Euclidean space $\mathbb{C}^{n}$ to $Y$ can be approximated, uniformly on $K$, by entire maps $\mathbb{C}^{n} \rightarrow Y$. If $X$ is a reduced Stein space and $\pi: Z \rightarrow X$ is a holomorphic fiber bundle with fiber $Y$ then we show that sections $X \rightarrow Z$ satisfy the Oka principle with approximation and interpolation. The analogous result is proved for sections of stratified fiber bundles, and of submersions with stratified sprays over Stein spaces.
\end{abstract}

Keywords: Stein spaces, fiber bundles, holomorphic mappings, Oka principle.

\section{INTRODUCTION}

In this paper we establish the Oka principle for sections of stratified holomorphic fiber bundles over Stein spaces under the condition that all fibers satisfy a Runge type approximation property (the convex approximation property, CAP) introduced in [14, 15], and also for sections of submersions with stratified sprays in the sense of Gromov. Our main results are Theorems 1.4, 6.5 and 8.3.

All complex spaces in this paper are assumed to be reduced and paracompact. For the general theory of Stein spaces we refer to [30].

Received May 8, 2007.

2000 Mathematics Subject Classification. 32E10, 32E30, 32H02

Supported by the research program P1-0291, Republic of Slovenia. 
We recall from [14] the definition of the Convex Approximation Property of a complex manifold. Let $z=\left(z_{1}, \ldots, z_{n}\right)$ be complex coordinates on $\mathbb{C}^{n}$, with $z_{j}=x_{j}+\mathrm{i} y_{j}$. Set

$$
Q=\left\{\left(z_{1}, \ldots, z_{n}\right) \in \mathbb{C}^{n}:\left|x_{j}\right| \leq 1,\left|y_{j}\right| \leq 1, j=1, \ldots, n\right\} .
$$

A special convex set in $\mathbb{C}^{n}$ is a compact convex set $K$ of the form

$$
K=\left\{z \in Q: y_{n} \leq h\left(z_{1}, \ldots, z_{n-1}, x_{n}\right)\right\},
$$

where $Q$ is the cube (1.1) and $h$ is a continuous concave function with values in $(-1,1)$. Such $(K, Q)$ will be called a special convex pair in $\mathbb{C}^{n}$.

Given a closed set $K$ in a complex space $X$, a map $f: K \rightarrow Z$ to a complex space $Z$ is said to be holomorphic if $f$ is the restriction to $K$ of a holomorphic map $U \rightarrow Z$ from an open neighborhood $U$ of $K$ in $X$. For a homotopy of maps the neighborhood $U$ will not depend on the parameter.

Definition 1.1. A complex manifold $Y$ satisfies the Convex Approximation Property (CAP) if for every special convex pair $(K, Q)$ in $\mathbb{C}^{n}(n \in \mathbb{N})$ and for every holomorphic map $f: K \rightarrow Y$ there is a sequence of holomorphic maps $f_{k}: Q \rightarrow Y$ $(k=1,2, \ldots)$ converging to $f$ uniformly on $K$.

By Theorem 1.4 below, CAP is equivalent to the condition that every holomorphic map $K \rightarrow Y$ from a compact convex set $K \subset \mathbb{C}^{n}(n \in \mathbb{N})$ can be approximated, uniformly on $K$, by entire maps $\mathbb{C}^{n} \rightarrow Y$.

Next we recall the notions of a holomorphic submersion and of a stratified holomorphic fiber bundle over a complex space.

Definition 1.2. Let $X$ and $Z$ be reduced complex spaces. A holomorphic map $\pi: Z \rightarrow X$ is a holomorphic submersion if for every point $z_{0} \in Z$ there exist an open neighborhood $V \subset Z$ of $z_{0}$, an open neighborhood $U \subset X$ of $x_{0}=\pi\left(z_{0}\right) \in$ $X$, an open set $W$ in $\mathbb{C}^{p}$, and a biholomorphic map $\phi: V \rightarrow U \times W$ such that $p r_{1} \circ \phi=\pi$, where $p r_{1}: U \times W \rightarrow U$ is the projection.

A stratification of a finite dimensional complex space $X$ is a finite descending chain of closed complex subvarieties of $X$,

$$
X=X_{0} \supset X_{1} \supset \cdots \supset X_{m}=\emptyset,
$$

such that each connected component $S$ (stratum) of any difference $X_{k} \backslash X_{k+1}$ is a complex manifold satisfying $\bar{S} \backslash S \subset X_{k+1}$ whose dimension $\operatorname{dim} S$ only depends on $k$. 
Definition 1.3. A holomorphic submersion $\pi: Z \rightarrow X$ (Def. 1.2) is a stratified holomorphic fiber bundle if $X$ admits a stratification (1.3) such that the restriction $\left.Z\right|_{S} \rightarrow S$ to every stratum $S \subset X_{k} \backslash X_{k+1}$ is a holomorphic fiber bundle over $S$.

Given a complex space $\left(X, \mathcal{O}_{X}\right)$, we denote by $\mathcal{O}(X)$ the algebra of all holomorphic functions on $X$. Recall that a compact set $K$ in $X$ is $\mathcal{O}(X)$-convex if for every point $p \in X \backslash K$ there exists an $f \in \mathcal{O}(X)$ such that $|f(p)|>\sup _{x \in K}|f(x)|$.

We are now ready to state our first main result which will also serve as the definition of various Oka-type properties; for the proof see $\S 5$.

Theorem 1.4. Assume that $X$ is a Stein space, that $\pi: Z \rightarrow X$ is a holomorphic submersion, and that $X$ is exhausted by a sequence of open relatively compact subsets $U_{1} \subset U_{2} \subset \cdots \subset \bigcup_{j=1}^{\infty} U_{j}=X$ such that each restriction $\left.Z\right|_{U_{j}} \rightarrow U_{j}$ is a stratified holomorphic fiber bundle all of whose fibers enjoy $\mathrm{CAP}$ (Def. 1.1). Choose a distance function $d$ on $Z$. Then the following hold:

(A) The basic Oka principle: Every continuous section $f: X \rightarrow Z$ of $\pi: Z \rightarrow X$ is homotopic to a holomorphic section.

(B) The basic Oka principle with interpolation and approximation: Given a continuous section $f: X \rightarrow Z$ that is holomorphic in a neighborhood of a compact $\mathcal{O}(X)$-convex subset $K$ in $X$ and whose restriction $\left.f\right|_{X^{\prime}}: X^{\prime} \rightarrow Z$ to a closed complex subvariety $X^{\prime}$ of $X$ is holomorphic on $X^{\prime}$, there exists for every $\epsilon>0$ a homotopy of continuous sections $f_{t}: X \rightarrow Z(t \in[0,1])$, with $f_{0}=f$, satisfying the following properties:

(i) $f_{1}$ is holomorphic on $X$,

(ii) $\left.f_{t}\right|_{X^{\prime}}=\left.f_{0}\right|_{X^{\prime}}$ for each $t \in[0,1]$, and

(iii) $f_{t}$ is holomorphic on $K$ and $\sup _{x \in K} d\left(f_{t}(x), f_{0}(x)\right)<\epsilon$ for each $t \in[0,1]$.

(C) The basic Oka principle with approximation and jet interpolation: Given $K$ and $X^{\prime}$ as in (B) and a continuous section $f: X \rightarrow Z$ that is holomorphic in an open set $V \supset K \cup X^{\prime}$, there exists for every $\ell \in \mathbb{N}$ and $\epsilon>0$ a homotopy $\left\{f_{t}\right\}_{t \in[0,1]}$ satisfying $(\mathrm{B})$ such that $f_{t}$ is holomorphic near $X^{\prime}$ and it agrees with $f=f_{0}$ to order $\ell$ along $X^{\prime}$ for every $t \in[0,1]$.

(D) The one-parametric Oka principle: Every homotopy $f_{t}: X \rightarrow Z(t \in[0,1])$ of sections between a pair of holomorphic sections $f_{0}, f_{1}$ can be deformed, with fixed ends at $t=0,1$, to a homotopy consisting of holomorphic sections. If the homotopy $f_{t}$ is fixed on a closed complex subvariety $X^{\prime}$ of $X$ then the deformation can be chosen fixed on $X^{\prime}$. 
Combining parts (A) and (D) in the conclusion of Theorem 1.4 we obtain the following corollary. (See also Corollary 6.8 below.)

Corollary 1.5. If $Z \rightarrow X$ is a stratified holomorphic fiber bundle over a Stein space $X$ such that all its fibers enjoy $\mathrm{CAP}$, then the inclusion

$$
\iota: \Gamma_{\mathcal{O}}(X, Z) \hookrightarrow \Gamma(X, Z)
$$

of the space of holomorphic sections into the space of continuous sections induces a bijection of the path connected components of the two spaces (endowed with the compact-open topology).

Many examples and sufficient conditions for the validity of CAP can be found in the papers $[14,15,16]$. In particular, if $Y$ admits a dominating holomorphic spray in the sense of Gromov [31] (such $Y$ is said to be elliptic), or, more generally, if it admits a finite dominating collection of holomorphic sprays (see [10]; such $Y$ is said to be subelliptic), then $Y$ satisfies CAP, and also its fully parametric version, PCAP (see Def. 6.4 below). The proof of this result is essentially a reduction to the Oka-Weil approximation theorem; see Theorem 4.1 in [21, p. 135] and Theorem 3.1 in [10, p. 534]. Hence Theorem 1.4 immediatelly implies the following corollary.

Corollary 1.6. If $\pi: Z \rightarrow X$ is a stratified holomorphic fiber bundle over a Stein space $X$ such that all its fibers are elliptic in the sense of Gromov [31], or subelliptic in the sense of [10], then sections $X \rightarrow Z$ satisfy the conclusions of Theorem 1.4.

We conclude this introduction by a brief survey of the known results on the Oka principle for section of holomorphic submersions.

The classical Oka-Grauert principle is essentially Theorem 1.4 in the special case when $\pi: Z \rightarrow X$ is a holomorphic principle bundle with a Lie group fiber, or an associated holomorphic fiber bundle with a complex homogeneous fiber. This was proved by Oka [42] for the Lie group $\mathbb{C}^{*}$ and by Grauert in general [28]. For expositions and extensions see Cartan [2], Grauert and Kerner [29], Forster and Ramspott [8, 9], Henkin and Leiterer [35], and Heinzner and Kutzschebauch [33]. Every complex homogeneous manifold satisfies CAP (see Grauert [26, 27]). Even for bundles with homogeneous fibers, Theorem 1.4 is stronger than Grauert's theorem since no conditions are imposed on the transition maps. 
The case of Theorem 1.4 when $X$ is a Stein manifold (without singularities) and $\pi: Z \rightarrow X$ is a holomorphic fiber bundle whose fiber $Y$ admits a dominating holomorphic spray is due to Gromov [31]. For exposition and extensions of Gromov's work see the papers [10,11, 21, 22, 23, 45]; for a homotopy theoretic point of view see Lárusson [38, 39, 40].

The CAP property was first introduced in [14] where Theorem 1.4 was proved in the case when $X$ is a Stein manifold and $X^{\prime}=\emptyset$, i.e., without the interpolation conditions (B-ii) and (C). Interpolation was added in [15, Theorem 1.1] (see also [40]). By [15, Corollary 1.3] (see also Lárusson [40]) the CAP property of a complex manifold $Y$ is equivalent to several ostensibly different Oka properties for maps of Stein manifolds to $Y$, expressed by the conditions (B-ii), (B-iii) and (C) in Theorem 1.4. The one-parametric Oka principle (part (D) in Theorem 1.4) is a simple consequence of the Oka principle with interpolation, (B-ii). (See the proof of Theorem 1.4 in $\S 5$.)

Although it was remarked in $[14,15]$ that Theorem 1.4 also holds when $X$ is a Stein space with singularities, a complete proof has not been available up to now. The outline proposed in [14, Remark 6.6, p. 705] requires certain not entirely obvious technical improvements in order to obtain the approximation statement. Here we give a complete proof, thereby dispelling any false impression that this is only a theory for manifolds.

We mention that the Oka principle furnished by Theorem 1.4 and Corollary 1.6 was used in the proof of embedding theorems for Stein spaces into Euclidean spaces of minimal dimension (see $[7,46,44]$ ). For embeddings with interpolation on discrete sequences see also [20,43].

In $\S 6$ we consider the parametric Oka principle; in this connection see also the remark at the very end of the paper.

In $\S 7$ we obtain existence results for holomorphic sections under suitable connectivity assumptions on the fibers.

Combining the induction scheme in this paper with the methods from $[22,23]$ we also obtain the Oka principle for sections of submersions with stratified sprays over Stein spaces (see Theorem 8.3 below). This result, which generalizes the work of Forster and Ramspott [9], was first stated by Gromov [31]. Complete details in the nonstratified case (i.e., for submersions with fiber dominating sprays over Stein manifolds) were given in [22]. A sketch of proof for the stratified case is found in $[23, \S 7]$, but without the approximation condition (only interpolation). 
The details in $\S 2-\S 5$ of this paper enable us to complete the proof of the full Oka principle as indicated in [23].

This general version of the Oka principle has recently been used by Ivarsson and Kutzschebauch [37] in a solution of the holomorphic Vaserstein problem posed by Gromov in [31].

Some recent extensions of the Oka principle should be mentioned: For sections of subelliptic holomorphic submersions over 1-convex manifolds (see Prezelj [45]); for sections of holomorphic Banach bundles over 1-convex manifolds (Leiterer and Vâjâitu [41]); and the soft Oka principle to the effect that the Oka principle holds universally if one allows homotopic deformations of the Stein structure on the source manifold (see [24, 25]).

In the course of proving Theorem 1.4 (see $\S 5$ ) we obtain some results of independent interest. In $\S 2$ we improve [15, Theorem 2.1] concerning the existence of open Stein neighborhoods of the set $K \cup X^{\prime}$ in Theorem 1.4. In $\S 3$ we obtain a parametric version of a theorem of Docquier and Grauert [6] on the existence of holomorphic retractions onto Stein submanifolds. In $\S 4$ we prove a semiglobal approximation/extension theorem that allows passage from a stratum to the next higher one.

\section{Open Stein neighborhoods}

The following result extends Theorem 2.1 from [15].

Theorem 2.1. Let $\left(X, \mathcal{O}_{X}\right)$ be a paracompact complex space, possibly nonreduced, and let $X^{\prime}$ be a closed Stein subvariety of $X$. Assume that $K$ is a compact set in $X$ that is $\mathcal{O}(\Omega)$-convex in an open Stein domain $\Omega \subset X$ containing $K$ and such that $K \cap X^{\prime}$ is $\mathcal{O}\left(X^{\prime}\right)$-convex. For every open set $U$ in $X$ containing $K \cup X^{\prime}$ there exists an open Stein domain $V$, with $K \cup X^{\prime} \subset V \subset U$, such that $K$ is $\mathcal{O}(V)$-convex.

The special case of Theorem 2.1 with $K=\emptyset$ is due to Siu [47] (see also Colţoiu [3] and Demailly [5]). The small improvement over [15, Theorem 2.1] is that Stein neighborhoods $V$ of $K \cup X^{\prime}$ can be chosen such that $K$ is $\mathcal{O}(V)$-convex. This property plays a key role in holomorphic approximations theorems, and hence it makes the result much more useful. Theorem 2.1 is used in $\S 4$ below to obtain a semiglobal approximation-interpolation result, Proposition 4.1, for sections of holomorphic submersions. 
As shown in [15], the necessity of $\mathcal{O}\left(X^{\prime}\right)$-convexity of $K \cap X^{\prime}$ in Theorem 2.1 is seen by taking

$$
X=\mathbb{C}^{2}, \quad X^{\prime}=\mathbb{C} \times\{0\}, \quad K=\left\{(z, w) \in \mathbb{C}^{2}: 1 \leq|z| \leq 2,|w| \leq 1\right\} .
$$

In this example, every Stein neighborhood of $K \cup X^{\prime}$ also contains the bidisc $\{(z, w):|z| \leq 2,|w| \leq 1\}$.

Proof of Theorem 2.1. A compact set $K$ that is $\mathcal{O}(\Omega)$-convex in an open Stein domain $\Omega \supset K$ in $X$ will be called holomorphically convex. By the classical theory this is equivalent to the existence of a plurisubharmonic function $\rho_{0}: \Omega \rightarrow \mathbb{R}_{+}$ such that $\rho_{0}^{-1}(0)=K$; we can choose $\rho_{0}$ to be smooth and strongly plurisubharmonic on $\Omega \backslash K$. (See [36, Theorem 5.1.5].) Fix a function $\rho_{0}$ with these properties.

Choose an open set $U \subset X$ containing $K \cup X^{\prime}$. As $X^{\prime}$ is Stein, the construction in [15, p. 737] gives an open set $W$ in $X$ such that $K \cup X^{\prime} \subset W \subset U$, a number $c>0$, and a smooth plurisubharmonic function $\rho: W \rightarrow \mathbb{R}_{+}$that agrees with $\rho_{0}$ in $U_{c}=\left\{x \in \Omega: \rho_{0}(x)<c\right\} \supset K$ and that satisfies $\rho>c$ on $W \backslash \bar{U}_{c}$. (See (i) and (ii) at the bottom of page 737 in [15].)

By [15, Theorem 2.1] there exists an open Stein domain $V$ in $X$ satisfying $K \cup X^{\prime} \subset V \subset W$. (In [15], the subvariety $X^{\prime}$ was denoted $X_{0}$. The ambient complex space $X$ was assumed to be reduced, but the latter property was never used in the proof. Compare with [5].) The restriction $\left.\rho\right|_{V}$ is a nonnegative plurisubharmonic function that vanishes precisely on $K$. Since $V$ is Stein, it follows that $K$ is $\mathcal{O}(V)$-convex.

\section{HOLOMORPHic RETRACTIONS}

The following well known result is obtained by combining a theorem of Docquier and Grauert ([6, Satz 3], [32, p. 257, Theorem 8]) with Siu's theorem on the existence of open Stein neighborhoods of Stein subvarieties [47, Corollary 1].

Theorem 3.1. Let $S$ be a Stein manifold, embedded as a locally closed complex submanifold in a complex manifold $M$. There exist an open Stein neighborhood $\Omega$ of $S$ in $M$ and a homotopy of holomorphic maps $\iota_{t}: \Omega \rightarrow \Omega(t \in[0,1])$ satisfying

(a) $\iota_{0}$ is the identity map on $\Omega$,

(b) $\left.\iota_{t}\right|_{S}$ is the identity map on $S$ for all $t \in[0,1]$, and

(c) $\iota_{1}(\Omega)=S$. 
The family $\left\{\iota_{t}\right\}_{t \in[0,1]}$ is a strong deformation retraction of $\Omega$ onto $S$ consisting of holomorphic mappings. There is no immediate analogue of Theorem 3.1 when $S$ is a Stein space with singularities.

We shall need the following parametric version of Theorem 3.1.

Proposition 3.2. Assume that $M$ and $B$ are finite dimensional complex spaces, $\pi: M \rightarrow B$ is a holomorphic submersion (Def. 1.2), and $S$ is a locally closed Stein subvariety of $M$ whose fiber $S_{b}=S \cap M_{b}(b \in B)$ is a locally closed complex submanifold of $M_{b}$ with dimension independent of $b \in B$. Then there exist an open Stein neighborhood $\Omega$ of $S$ in $M$ and a homotopy of holomorphic maps $\iota_{t}: \Omega \rightarrow \Omega(t \in[0,1])$ satisfying properties $(a)-(c)$ in Theorem 3.1 and such that $\pi \circ \iota_{t}=\pi$ for each $t \in[0,1]$.

Proof. We begin by recalling the proof in the classical case when $B$ is a singleton. Now $S$ is a locally closed Stein submanifold of a complex manifold $M$. By Theorem 2.1 we can replace $M$ by an open Stein neighborhood of $S$ that contains $S$ as a closed submanifold. We consider the holomorphic tangent bundle $T S$ as a subbundle of $\left.T M\right|_{S}$, making the usual identification of $T M$ with $T^{(1,0)} M$. By Cartan's Theorem A there exist finitely many holomorphic vector fields $V_{1}, \cdots, V_{N}$ on $M$ such that the vectors $V_{1}(x), \ldots, V_{N}(x)$ span $T_{x} M$ over $\mathbb{C}$ at every point $x \in M$. Let $\phi_{t}^{j}$ denote the flow of $V_{j}$, i.e., the solution of the ordinary differential equation

$$
\dot{\phi}_{t}^{j}(x)=V_{j}\left(\phi_{t}^{j}(x)\right), \quad \phi_{0}^{j}(x)=x .
$$

For each point $x \in M$ there is a number $T=T(x)>0$ such that $\phi_{t}^{j}(x)$ exists for all $t \in \mathbb{C}$ in the disc $|t|<T$, and $T$ can be chosen independent of the point $x$ belonging to a compact subset of $M$. The map

$$
F\left(x, t_{1}, \ldots, t_{N}\right)=\phi_{t_{1}}^{1} \circ \cdots \circ \phi_{t_{N}}^{N}(x), \quad x \in S, t_{1}, \ldots, t_{N} \in \mathbb{C}
$$

is defined and holomorphic in an open neighborhood of $S \times\{0\}^{N}$ in $S \times \mathbb{C}^{N}$. We have $F(x, 0)=x$ and $\left.\frac{\partial}{\partial t_{j}} F(x, t)\right|_{t=0}=V_{j}(x)$ for every $x \in S$. As the $V_{j}$ 's span $T M$ at every point, $F$ is a submersion along $S \times\{0\}^{N}$. Let

$$
\Theta_{x}=\left.\partial_{t}\right|_{t=0} F(x, t): \mathbb{C}^{N} \rightarrow T_{x} M, \quad x \in S .
$$

The subset $E \subset S \times \mathbb{C}^{N}$ with the fibers

$$
E_{x}=\left\{v \in \mathbb{C}^{N}: \Theta_{x}(v) \in T_{x} S\right\}, \quad x \in S
$$


is then a holomorphic vector subbundle of the trivial bundle $S \times \mathbb{C}^{N}$. Since $S$ is Stein, there is a holomorphic vector subbundle $\nu \subset S \times \mathbb{C}^{N}$ such that $S \times \mathbb{C}^{N}=E \oplus \nu\left[32\right.$, Theorem 7, p. 256]. By the construction, $\Theta:\left.\nu \rightarrow T M\right|_{S}$ is an injective holomorphic vector bundle map and $\left.T M\right|_{S}=T S \oplus \Theta(\nu)$; thus $\nu \cong \Theta(\nu)$ is the normal bundle of $S$ in $M$.

Consider the restriction of $F$ to $\nu$. By the inverse function theorem $F$ maps an open neighborhood $\Omega^{\prime}$ of the zero section in $\nu$ biholomorphically onto an open neighborhood $\Omega=F\left(\Omega^{\prime}\right)$ of $S$ in $M$. Further, choosing $\Omega^{\prime}$ to have convex fibers, $F$ conjugates the family of radial dilations $\iota_{t}^{\prime}(v)=(1-t) v\left(v \in \Omega^{\prime}, t \in[0,1]\right)$ to a family of holomorphic maps $\iota_{t}: \Omega \rightarrow \Omega$ satisfying the stated properties. (It is possible to choose $\Omega^{\prime}$ Stein and with convex fibers: take $\Omega_{x}^{\prime}=\left\{v \in \nu_{x}: e^{\phi(x)}|v|^{2}<\right.$ 1) where $\phi: S \rightarrow \mathbb{R}_{+}$is a sufficiently fast growing strongly plurisubharmonic function and $|v|$ is the Euclidean norm of the vector $v \in \nu_{x} \subset \mathbb{C}^{N}$.)

The above proof extends to the general case: Let $V T(M) \rightarrow M$ denote the vertical tangent bundle of $M$, consisting of all tangent vector to the (regular) fibers $M_{b}=\pi^{-1}(b), b \in B$. As before we replace $M$ by an open Stein domain containing $S$ as a closed Stein subspace. Then $S_{b}=S \cap M_{b}$ is a closed Stein submanifold of $M_{b}$ for every $b \in B$, and $V T(S)$ is a holomorphic vector subbundle of $\left.V T(M)\right|_{S}$. Select finitely many holomorphic sections $V_{1}, \ldots, V_{N}$ of $V T(M)$ that generate the latter bundle at each point of $M$. (This is possible by a generalization of Cartan' Theorem A, using an induction on the dimension of the exceptional set where the sections fail to generate.) The flow $\phi_{t}^{j}$ of $V_{j}$ is well defined and holomorphic in a neighborhood of $M \times\{0\}$ in $M \times \mathbb{C}$, and it remains in the fibers of $\pi$. We can now complete the proof exactly as before by splitting $S \times \mathbb{C}^{N}=E \oplus \nu$ and restricting the map $F(3.1)$ to a suitable Stein neighborhood of the zero section in $\nu$.

\section{A HolOMORPHiC APPROXIMATION-INTERPOLATION THEOREM}

In this section we prove the following result which will enable us to pass from one stratum to the next one in the proof of Theorem 1.4.

Proposition 4.1. Assume that $X^{\prime}$ is a closed Stein subvariety of a complex space $X$ and that $K$ is a compact holomorphically convex set in $X$ (as in Theorem 2.1) such that $K \cap X^{\prime}$ is $\mathcal{O}\left(X^{\prime}\right)$-convex. Let $\pi: Z \rightarrow X$ be a holomorphic submersion of a complex space $Z$ onto $X$ (Def. 1.2). Assume that $U \subset X$ is an open set 
containing $K$ and $f:\left.U \cup X^{\prime} \rightarrow Z\right|_{U \cup X^{\prime}}$ is a section whose restrictions to $U$ and to $X^{\prime}$ are holomorphic. Then there exist open Stein neighborhoods $V_{j}$ of $K \cup X^{\prime}$ in $X$ and holomorphic sections $f_{j}:\left.V_{j} \rightarrow Z\right|_{V_{j}}(j=1,2, \ldots)$ such that $\left.f_{j}\right|_{X^{\prime}}=\left.f\right|_{X^{\prime}}$ for all $j \in \mathbb{N}$ and $\left.\lim _{j \rightarrow \infty} f_{j}\right|_{K}=\left.f\right|_{K}$ (the convergence is uniform on $K$ ).

When $X$ is a Stein manifold, $K$ is $\mathcal{O}(X)$-convex, and $f: U \cup X^{\prime} \rightarrow Z$ is a holomorphic map to a complex manifold $Z$, this is [15, Theorem 3.1].

Proof. It suffices to show that for every compact $\mathcal{O}(X)$-convex set $L \subset X$ there is a sequence of sections $f_{j}$ in open neighborhoods $V_{j}$ of $K \cup\left(L \cap X^{\prime}\right)$ satisfying the conclusion of Proposition 4.1; the result then follows by an induction over a normal exhaustion of $X$. Hence we may assume that $X$ is finite dimensional.

Further, by Theorem 2.1 we can replace $X$ by an open Stein neighborhood of $K \cup X^{\prime}$ such that $K$ is $\mathcal{O}(X)$-convex, and we can choose the neighborhood $U$ of $K$ to be Stein and relatively compact in $X$. Since $K$ is $\mathcal{O}(X)$-convex, it is also $\mathcal{O}(U)$-convex.

We begin by considering the case when $Z=X \times \mathbb{C}^{p}$ and $\pi: Z \rightarrow X$ is the projection $\pi(x, \zeta)=x$. We identify sections $X \rightarrow Z$ with maps $f: X \rightarrow \mathbb{C}^{p}$ to the fiber. By Cartan's extension theorem there is a holomorphic map $\phi: X \rightarrow \mathbb{C}^{p}$ such that $\left.\phi\right|_{X^{\prime}}=\left.f\right|_{X^{\prime}}$. There exist finitely many functions $h_{1}, \ldots, h_{m} \in \mathcal{O}(X)$ that vanish on the subvariety $X^{\prime}$ and that generate the ideal sheaf of $X^{\prime}$ at every point of $U \Subset X$. Since $U$ is Stein, Cartan's Theorem B furnishes holomorphic maps $g_{k}: U \rightarrow \mathbb{C}^{p}(k=1, \ldots, m)$ such that $f=\phi+\sum_{k=1}^{m} g_{k} h_{k}$ on $U$. By the Oka-Weil theorem $g_{k}$ is a uniform limit on $K$ of a sequence of holomorphic maps $g_{k, j}: X \rightarrow \mathbb{C}^{p}(j=1,2, \ldots)$. The sequence of holomorphic maps

$$
f_{j}=\phi+\sum_{k=1}^{m} g_{k, j} h_{k}: X \rightarrow \mathbb{C}^{p}
$$

then satisfies Proposition 4.1 in this special case.

We now turn to the general case. Consider the following subsets of $Z$ :

$$
\widetilde{K}=f(K), \quad \tilde{X}^{\prime}=f\left(X^{\prime}\right), \quad \widetilde{U}=f(U) .
$$

Since $f: U \rightarrow \widetilde{U}$ is biholomorphic, $\widetilde{U}$ is Stein. Theorem 2.1 furnishes an open Stein set $\widetilde{\Omega}$ in $Z$ containing $\widetilde{U}$ as a closed subvariety. As $K$ is $\mathcal{O}(U)$-convex, we infer that $\widetilde{K}$ is $\mathcal{O}(\widetilde{U})$-convex, and hence $\mathcal{O}(\widetilde{\Omega})$-convex. Since $X^{\prime}$ is Stein and $K \cap X^{\prime}$ is $\mathcal{O}\left(X^{\prime}\right)$-convex, $\widetilde{X}^{\prime}$ is a Stein subvariety of $Z$ and $\widetilde{K} \cap \widetilde{X}^{\prime}=f\left(K \cap X^{\prime}\right)$ is $\mathcal{O}\left(\widetilde{X}^{\prime}\right)$-convex. Theorem 2.1 now shows that 
(*) $\widetilde{K} \cup \widetilde{X}^{\prime}$ admits a basis of Stein neighborhoods in $Z$.

We shall need the following Lemma.

Lemma 4.2. There exist an open Stein neighborhood $W$ of $\widetilde{K} \cup \widetilde{X}^{\prime}$ in $Z$ and a holomorphic embedding $G: W \hookrightarrow X \times \mathbb{C}^{N}$ for some $N \in \mathbb{N}$ such that for every $x \in X, G$ embeds the fiber $W_{x}=W \cap \pi^{-1}(x)$ onto a locally closed Stein submanifold $G\left(W_{x}\right)$ of $\{x\} \times \mathbb{C}^{N}$. ( $W_{x}$ may be empty for some $x \in X$.)

Proof. Since the fibers $Z_{x}$ are smooth and of constant dimension $p$, the vertical tangent bundle $V T(Z)$, consisting of all tangent vectors to the fibers $Z_{x}$, is a holomorphic vector bundle of rank $p$ over $Z$. For each holomorphic function $g$ on an open subset of $Z$ we denote by $V d(g)$ the differential of $g$ in the vertical directions $V T(Z)$; hence $V d(g)$ is a holomorphic section of the vertical cotangent bundle $V T^{*}(Z)$.

Let $W_{0} \Subset Z$ be a Stein open neighborhood of $\widetilde{K} \cup \widetilde{X}^{\prime}$ furnished by $(*)$. By Cartan's Theorem A there exist finitely many functions $g_{1}, \ldots, g_{N} \in \mathcal{O}\left(W_{0}\right)$ whose vertical differentials $V d\left(g_{j}\right)$ span the vertical cotangent space $V T_{z}^{*}(Z)$ at each point $z \in W_{0}$. Consider the holomorphic map

$$
G: W_{0} \rightarrow X \times \mathbb{C}^{N}, \quad G(z)=\left(\pi(z), g_{1}(z), \ldots, g_{N}(z)\right) .
$$

Our choice of the $g_{j}$ 's implies that $G$ embeds an open neighborhood $W \subset W_{0}$ of $\widetilde{K} \cup \widetilde{X}^{\prime}$ biholomorphically onto a locally closed complex subvariety $G(W)$ of $X \times \mathbb{C}^{N}$. Clearly we have $p r_{1} \circ G=\pi$, where $p r_{1}: X \times \mathbb{C}^{N} \rightarrow X$ is the projection $\operatorname{pr}_{1}(x, \zeta)=x$, and $G\left(W_{x}\right)$ is a locally closed complex submanifold (without singularities) of $\{x\} \times \mathbb{C}^{N}$ for every $x$. By $\left(^{*}\right.$ ) we may choose $W$ Stein, and then each fiber $W_{x}$ is also Stein.

We now complete the proof of Proposition 4.1. Let $G: W \hookrightarrow X \times \mathbb{C}^{N}$ be a holomorphic embedding furnished by Lemma 4.2. Proposition 3.2, applied to the Stein subvariety $S=G(W)$ in $X \times \mathbb{C}^{N}$ with regular fibers $S_{x}=G\left(W_{x}\right) \subset\{x\} \times$ $\mathbb{C}^{N}$, gives an open Stein neighborhood $\Omega \subset X \times \mathbb{C}^{N}$ of $S$ and a fiber preserving holomorphic retraction $\iota: \Omega \rightarrow S$ which retracts each fiber $\Omega_{x}=\Omega \cap\left(\{x\} \times \mathbb{C}^{N}\right)$ onto the fiber $S_{x}$. After shrinking $U$ around $K$ we may assume that $f(U) \subset W$. Consider the composed section

$$
G \circ f: U \cup X^{\prime} \rightarrow\left(U \cup X^{\prime}\right) \times \mathbb{C}^{N} \subset X \times \mathbb{C}^{N} .
$$


By the special case proved above there is a sequence of holomorphic sections $F_{j}: V_{j} \rightarrow V_{j} \times \mathbb{C}^{N}(j=1,2, \ldots)$ in open sets $V_{j} \supset K \cup X^{\prime}$ such that $\left.F_{j}\right|_{X^{\prime}}=$ $\left.G \circ f\right|_{X^{\prime}}$ for each $j$, and $\left.\lim _{j \rightarrow \infty} F_{j}\right|_{K}=\left.G \circ f\right|_{K}$ uniformly on $K$. For sufficiently large $j$, and for $V_{j} \supset K \cup X^{\prime}$ chosen small enough, we have that $F_{j}\left(V_{j}\right) \subset \Omega$ (the domain of the retraction $\iota$ ). The sequence of holomorphic sections

$$
f_{j}=G^{-1} \circ \iota \circ F_{j}:\left.V_{j} \rightarrow Z\right|_{V_{j}}, \quad j=1,2, \ldots
$$

then fulfills Proposition 4.1.

Remark 4.3. Our proof gives the following addition to Proposition 4.1. Assume that $f$ satisfies the hypotheses of the proposition and that $\phi:\left.W \rightarrow Z\right|_{W}$ is a holomorphic section in an open neighborhood $W$ of $X^{\prime}$ such that $f=\phi$ on $X^{\prime}$, and such that $f$ and $\phi$ are tangent to order $\ell \in \mathbb{N}$ along $K \cap X^{\prime}$. Then the sequence $\left\{f_{j}\right\}$ in Proposition 4.1 can be chosen such that, in addition to the stated properties, $f_{j}$ is tangent to $\phi$ to order $\ell$ along $X^{\prime}$ for each $j=1,2, \ldots$.

This follows from the setup (4.1) by choosing $h_{1}, \ldots, h_{m} \in \mathcal{O}(X)$ that vanish to order $\ell$ on the subvariety $X^{\prime}$ and that generate the $\ell$-th power of the ideal sheaf of $X^{\prime} \subset X$ at every point of the compact set $K$.

Remark 4.4. Proposition 4.1 extends to continuous families of sections with a parameter in a compact Hausdorff space.

As a simple case, let us consider a homotopy of sections $f_{t}: U \cup X^{\prime} \rightarrow Z$ for $t \in[0,1]$ such that each $f_{t}$ satisfies the conditions in the proposition and the homotopy is fixed on $X^{\prime}$. (Here $U$ is an open set in $X$ containing a compact $\mathcal{O}(X)$-convex set $K$.) It is then possible to find another homotopy $g_{t}: V \rightarrow Z$ $(t \in[0,1])$, consisting of sections that are holomorphic in a neighborhood $V$ of $K \cup X^{\prime}$, such that $g_{t}=f_{t}=f_{0}$ on $X^{\prime}$ and $g_{t}$ approximates $f_{t}$ uniformly on $K$ as close as desired for every $t \in[0,1]$. In addition, we can choose $g_{t}$ tangent to order $\ell \in \mathbb{N}$ along $X^{\prime}$ to a chosen holomorphic extension $\phi$ of $\left.f_{0}\right|_{X^{\prime}}$.

This extension is easy to prove for the trivial fibration $Z=X \times \mathbb{C}^{p} \rightarrow X$. In the general case, a Stein neighborhood of $f_{t}\left(K \cup X^{\prime}\right)$ in $Z$ is used to get a corresponding approximating section $g_{t}$ for a fixed $t \in[0,1]$, and hence a patching problem appears when trying to find a family $g_{t}$ depending continuously on $t \in$ $[0,1]$. This problem is solved by the method of successive patching explained in [21, p. 139]. 


\section{Proof of Theorem 1.4}

The proof of Theorem 1.4 proceeds by a double induction: The outer one over an exhaustion of $X$ by compact holomorphically convex sets, and the inner one over the strata in a suitable stratification. The main step for the latter induction is given by the following proposition.

Proposition 5.1. Assume that $X$ is a Stein space, $M_{1} \subset M_{0}$ are closed complex subvarieties of $X$ such that $S=M_{0} \backslash M_{1}$ is a complex manifold, and $\pi: Z \rightarrow X$ is a holomorphic submersion such that $\left.Z\right|_{S} \rightarrow S$ is a holomorphic fiber bundle whose fiber enjoys CAP. Let $d$ be a complete distance function on $Z$. Given a pair of compact $\mathcal{O}(X)$-convex subsets $K \subset L$ of $X$ and a continuous section $f: X \rightarrow Z$ that is holomorphic in an open neighborhood of $K_{1}=K \cup\left(L \cap M_{1}\right)$, there exists for every $\epsilon>0$ and $\ell \in \mathbb{N}$ a homotopy of continuous sections $f_{t}: X \rightarrow Z(t \in$ $[0,1])$ that are holomorphic in a neighborhood of $K_{1}$, with $f_{0}=f$, satisfying the following:

(i) $f_{t}$ agrees with $f_{0}$ to order $\ell$ along $M_{1} \cap L$ for each $t \in[0,1]$,

(ii) $\sup _{x \in K, t \in[0,1]} d\left(f_{t}(x), f_{0}(x)\right)<\epsilon$, and

(iii) $f_{1}$ is holomorphic in a neighborhood of $K_{0}=K \cup\left(L \cap M_{0}\right)$ in $X$.

Proof of Theorem 1.4. Assume Proposition 5.1 for the moment. Choose a sequence of compact $\mathcal{O}(X)$-convex sets

$$
K=K_{0} \subset K_{1} \subset K_{2} \subset \cdots \subset \bigcup_{k=0}^{\infty} K_{k}=X .
$$

Set $t_{k}=1-2^{-k}$ and $I_{k}=\left[t_{k}, t_{k+1}\right]$ for $k=0,1, \ldots$; hence $\cup_{k=0}^{\infty} I_{k}=[0,1)$.

Let $f=f_{0}: X \rightarrow Z$ be a continuous section that is holomorphic on a complex subvariety $X^{\prime}$ of $X$ and in an open neighborhood of $K=K_{0}$ in $X$. Given $\epsilon>0$, we need to find a homotopy of sections $f_{t}: X \rightarrow Z(t \in[0,1])$ satisfying the conclusion (B) in Theorem 1.4. Essentially the same proof will give the conclusion (C) if $f_{0}$ is holomorphic in a neighborhood of $K \cup X^{\prime}$.

By induction on $k \in \mathbb{Z}_{+}$we shall find a sequence of homotopies of sections $f_{t}: X \rightarrow Z\left(t \in I_{k}\right)$ that agree at the common endpoint $t_{k+1}$ of the adjacent intervals $I_{k}, I_{k+1}$ and such that the following hold: 
(i) for every $k=0,1, \ldots$ and $t \in I_{k}$ the section $f_{t}$ is holomorphic in an open neighborhood of $K_{k}$ and satisfies

$$
\sup _{x \in K_{k}} d\left(f_{t}(x), f_{t_{k}}(x)\right)<2^{-k-1} \epsilon,
$$

(ii) the homotopy $\left\{f_{t}: t \in[0,1)\right\}$ is fixed on the subvariety $X^{\prime}$.

These properties clearly imply that the limit section

$$
f_{1}=\lim _{t \rightarrow 1} f_{t}: X \rightarrow Z
$$

exists and is holomorphic on $X, \sup _{x \in K_{0}} d\left(f_{1}(x), f_{0}(x)\right)<\epsilon$, and $\left.f_{1}\right|_{X^{\prime}}=\left.f_{0}\right|_{X^{\prime}}$. Thus the homotopy $\left\{f_{t}\right\}_{t \in[0,1]}$ satisfies the required properties.

Since all steps in the induction are of the same kind, we explain how to get the first homotopy for $t \in I_{0}=\left[0, \frac{1}{2}\right]$.

Set $K=K_{0}, L=K_{1}$. By the assumption there exists an open set $U \Subset X$ containing $L$ such that $\left.Z\right|_{U}$ is a stratified holomorphic fiber bundle whose strata satisfy CAP. Since $L$ is $\mathcal{O}(X)$-convex, there is a Stein domain $\Omega$ in $X$ with $L \subset \Omega \Subset U$. Then $\Omega$ is a finite dimensional Stein space and the restriction $\left.Z\right|_{\Omega} \rightarrow \Omega$ is also a stratified fiber bundle all of whose fibers enjoy CAP. Choose a stratification

$$
\Omega=X_{0} \supset X_{1} \supset \cdots \supset X_{m}=\emptyset
$$

such that the restriction of $\pi: Z \rightarrow X$ to each stratum $S \subset X_{k} \backslash X_{k+1}$ is a fiber bundle whose fiber enjoys CAP. Taking $X_{k}^{\prime}=X_{k} \cup\left(X^{\prime} \cap \Omega\right)$ gives another stratification

$$
\Omega=X_{0}^{\prime} \supset X_{1}^{\prime} \supset \cdots \supset X_{m}^{\prime}=\Omega \cap X^{\prime},
$$

with regular strata $X_{k}^{\prime} \backslash X_{k+1}^{\prime}=X_{k} \backslash\left(X_{k+1} \cup X^{\prime}\right) \subset X_{k} \backslash X_{k+1}$, ending with $X_{m}^{\prime}=$ $\Omega \cap X^{\prime}$.

By Proposition 4.1 we can replace $f_{0}$ by a section that is holomorphic in an open neighborhood of $K \cup\left(L \cap X^{\prime}\right)$. When proving (C), $f_{0}$ is already assumed to be holomorphic near $X^{\prime}$.

Let $\left\{f_{t}\right\}_{t \in\left[0, \frac{1}{2 m}\right]}$ be a homotopy furnished by Proposition 5.1 for the pair of subvarieties $M_{1}=X_{m}^{\prime}=\Omega \cap X^{\prime}$ and $M_{0}=X_{m-1}^{\prime}$ of $\Omega$, with $\epsilon$ replaced by $\frac{\epsilon}{2 m}$. Then $f_{\frac{1}{2 m}}$ is holomorphic in a neighborhood of $K \cup\left(L \cap X_{m-1}^{\prime}\right)$, the homotopy is fixed on $X^{\prime}$, and it satisfies

$$
\sup _{x \in K_{0}} d\left(f_{t}(x), f_{0}(x)\right)<\frac{\epsilon}{2 m}, \quad t \in\left[0, \frac{1}{2 m}\right] .
$$


Next we apply Proposition 5.1 with the initial section $f=f_{\frac{1}{2 m}}$ and the pair of subvarieties $M_{1}=X_{m-1}^{\prime}, M_{0}=X_{m-2}^{\prime}$ to get a homotopy $\left\{f_{t}\right\}_{t \in\left[\frac{1}{2 m}, \frac{2}{2 m}\right]}^{\frac{1}{2 m}}$ that is fixed on $X^{\prime}$ such that the section $f_{\frac{2}{2 m}}=f_{\frac{1}{m}}$ is holomorphic in a neighborhood of $K \cup\left(L \cap X_{m-2}^{\prime}\right)$, and such that

$$
\sup _{x \in K_{0}} d\left(f_{t}(x), f_{\frac{1}{2 m}}(x)\right)<\frac{\epsilon}{2 m}, \quad t \in\left[\frac{1}{2 m}, \frac{2}{2 m}\right] .
$$

Continuing in this way we obtain after $m$ steps a homotopy $\left\{f_{t}\right\}_{t \in\left[0, \frac{1}{2}\right]}$ with the required properties. In particular, the section $f_{\frac{1}{2}}$ is holomorphic in a neighborhood of the set $L=K_{1}$, and its restriction to the subvariety $X^{\prime}$ agrees with $\left.f_{0}\right|_{X^{\prime}}$. We can extend this homotopy to all of $X$ (without changing it near $L=K_{1}$ ) by using a cut-off function in the parameter.

In the same way we construct homotopies $\left\{f_{t}\right\}_{t \in I_{k}}$ for all $k=1,2, \ldots$, thereby proving part (B).

When the initial section $f=f_{0}$ is holomorphic in a neighborhood of $K \cup X^{\prime}$, we can use the improvements of Proposition 4.1 in Remarks 4.3 and 4.4 to keep the sections $f_{t}$ in our homotopy holomorphic in a neighborhood of $K \cup X^{\prime}$ and tangent to $f_{0}$ to order $\ell$ along $X^{\prime}$. This gives the conclusion $(\mathrm{C})$.

Finally we show that Theorem 1.4 (D) is a consequence of the interpolation statement (B-ii). Suppose that $f_{0}, f_{1} \in \Gamma_{\mathcal{O}}(X, Z)$ are connected by a homotopy $\left\{f_{t}\right\}_{t \in[0,1]} \subset \Gamma(X, Z)$ that is fixed on a closed complex subvariety $X^{\prime}$ of $X$ (possibly empty). Choose a retraction $\theta$ of $\mathbb{C}$ onto the segment $[0,1] \subset \mathbb{C}$ and define $F: \widetilde{X}=X \times \mathbb{C} \rightarrow \widetilde{Z}=Z \times \mathbb{C}$ by

$$
F(x, t)=\left(f_{\theta(t)}(x), t\right), \quad x \in X, t \in \mathbb{C} .
$$

Then $F$ is a section of the fiber bundle $\tilde{\pi}: \widetilde{Z} \rightarrow \widetilde{X}$ with the fiber $Y$ and the projection map $\widetilde{\pi}(z, t)=(\pi(z), t)$. Since $F$ is holomorphic on the subvariety

$$
\tilde{X}^{\prime}=(X \times\{0,1\}) \cup\left(X^{\prime} \times \mathbb{C}\right) \subset \tilde{X},
$$

part (B) of Theorem 1.4 furnishes a homotopy that is fixed on $\tilde{X}^{\prime}$ from $F$ to a holomorphic section $\widetilde{F}: \widetilde{X} \rightarrow \widetilde{Z}$. The family $\widetilde{f}_{t}: X \rightarrow Z$, defined by

$$
\widetilde{F}(x, t)=\left(\widetilde{f}_{t}(x), t\right), \quad x \in X, t \in[0,1],
$$

is then a homotopy of holomorphic sections of $\pi: Z \rightarrow X$ such that $\widetilde{f}_{0}=f_{0}$, $\widetilde{f}_{1}=f_{1}$, and $\left.\widetilde{f}_{t}\right|_{X^{\prime}}=\left.f_{t}\right|_{X^{\prime}}$ for all $t \in[0,1]$.

This proves Theorem 1.4 provided that Proposition 5.1 holds. 
Proof of Proposition 5.1. When $X$ is smooth (without singularities) and $M_{0}=$ $X$, this is [15, Proposition 4.1]. We first recall the proof of this special case since the general case will be obtained by a modification explained below.

We may assume that $L=\{x \in X: \rho(x) \leq 0\}$, where $\rho: X \rightarrow \mathbb{R}$ is a strongly plurisubharmonic exhaustion function such that $\left.\rho\right|_{K}<0$ and $d \rho \neq 0$ on $b L=$ $\{\rho=0\}$.

We recall the geometric setup from $[12, \S 6.5]$ that was also used in $[15, \S 4]$. By the assumption, $f$ is holomorphic in an open set $U \supset K \cup M_{1}$. Since the compact set $K_{1}=K \cup\left(M_{1} \cap L\right) \subset U$ is $\mathcal{O}(X)$-convex, there exists a smooth strongly plurisubharmonic exhaustion function $\tau: X \rightarrow \mathbb{R}$ such that $\tau<0$ on $K_{1}$ and $\tau>0$ on $X \backslash U$. By general position we may assume that 0 is a regular value of $\tau$ and the hypersurfaces $\{\rho=0\}=b L$ and $\{\tau=0\}$ intersect transversely along the real codimension two submanifold $\Sigma=\{\rho=0\} \cap\{\tau=0\}$. Hence $D_{0}:=\{\tau \leq 0\} \subset U$ is a strongly pseudoconvex domain with smooth boundary. For each $s \in[0,1]$ let

$$
\rho_{s}=\tau+s(\rho-\tau)=(1-s) \tau+s \rho, \quad D_{s}=\left\{\rho_{s} \leq 0\right\}=\{\tau \leq s(\tau-\rho)\} .
$$

We have $D_{0}=\{\tau \leq 0\}$ and $D_{1}=\{\rho \leq 0\}=L$.

Let $\Omega=\{\rho<0, \tau>0\} \subset D_{1} \backslash D_{0}$ and $\Omega^{\prime}=\{\rho>0, \tau<0\} \subset D_{0} \backslash D_{1}$ (see Figure 1). As $s$ increases from 0 to $1, D_{s} \cap L$ increases to $D_{1}=L$ while $D_{s} \backslash L \subset D_{0}$ decreases to $\emptyset$. All hypersurfaces $b D_{s}=\left\{\rho_{s}=0\right\}$ intersect along $\Sigma$. Since $d \rho_{s}=(1-s) d \tau+s d \rho$ and the differentials $d \tau, d \rho$ are linearly independent along $\Sigma, b D_{s}$ is smooth near $\Sigma$. Finally, $b D_{s}$ is strongly pseudoconvex at every smooth point, in particular at every point where $d \rho_{s} \neq 0$.

We investigate the singular points of $b D_{s}=\left\{\rho_{s}=0\right\}$ inside $\Omega$. (The remaining singular points will be irrelevant.) The defining equation of $D_{s} \cap \Omega$ can be written as $\tau \leq s(\tau-\rho)$ and, after dividing by $\tau-\rho>0$, as

$$
D_{s} \cap \Omega=\left\{x \in \Omega: h(x)=\frac{\tau(x)}{\tau(x)-\rho(x)} \leq s\right\} .
$$

The critical point equation $d h=0$ is equivalent to

$$
(\tau-\rho) d \tau-\tau(d \tau-d \rho)=\tau d \rho-\rho d \tau=0 .
$$

A generic choice of $\rho$ and $\tau$ insures that there are at most finitely many solutions $p_{1}, \ldots, p_{m} \in \Omega$ and no solution on $b \Omega$. A calculation shows that at each critical point the complex Hessians satisfy $(\tau-\rho)^{2} H_{h}=\tau H_{\rho}-\rho H_{\tau}$. Since $\tau>0$ and 


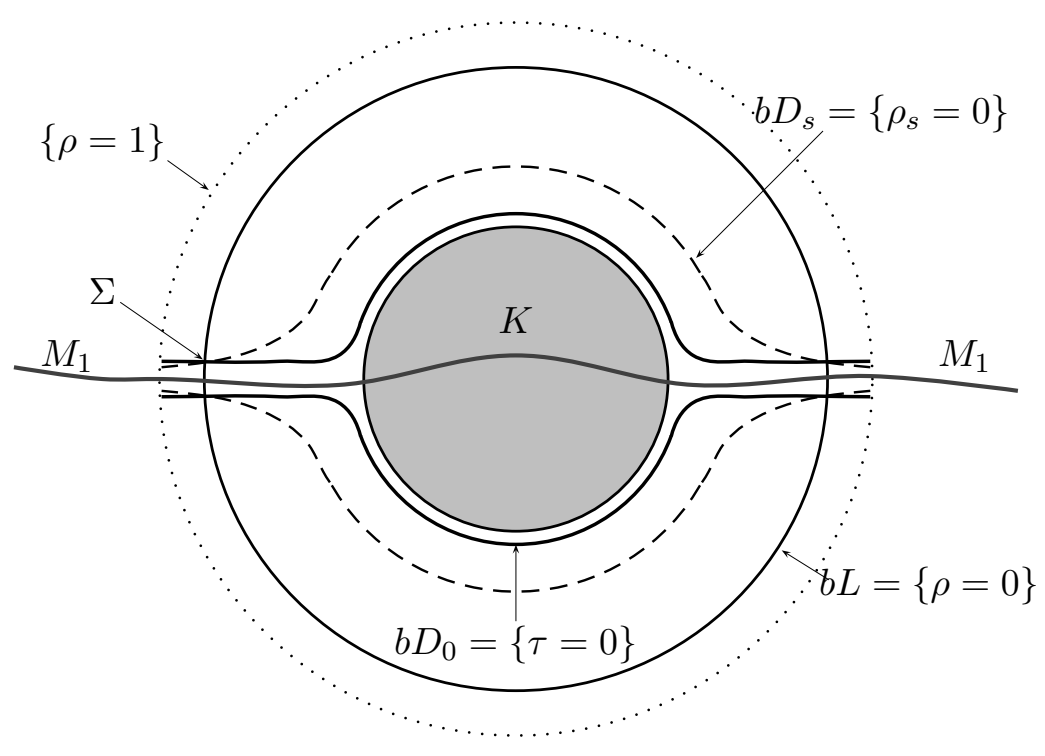

Figure 1. The sets $D_{s}$.

$-\rho>0$ on $\Omega$, we conclude that $H_{h}>0$ at such points. We may assume that distinct critical points of $h$ belong to different level sets.

We are now ready to prove Proposition 5.1 in the special case. Let $s_{0}$ and $s_{1}$ be two regular values of $h$ on $\Omega$ such that $0 \leq s_{0}<s_{1} \leq 1$ and $h$ has at most one critical point in $\Omega_{s_{0}, s_{1}}=\left\{x \in \Omega: s_{0}<h(x)<s_{1}\right\}$. Suppose inductively that we have already found a homotopy $f_{t}: X \rightarrow Z, t \in\left[0, s_{0}\right]$, satisfying the conditions in the proposition and such that $f_{s_{0}}$ is holomorphic in a neighborhood of $D_{s_{0}}$. We wish to deform $f_{s_{0}}$ to a section $f_{s_{1}}$ that is holomorphic in a neighborhood of $D_{s_{1}}$ by a homotopy consisting of sections that are holomorphic near $D_{s_{0}} \cap D_{s_{1}}$ and such that the homotopy is fixed on $M_{1}$. We consider two cases.

The noncritical case: $h$ has no critical values in $\Omega_{s_{0}, s_{1}}$.

Recall that a pair $(A, B)$ of compact sets in a complex space $X$ is said to be a Cartan pair if the following hold (see [14, p. 695]):

(i) $A \cup B$ and $A \cap B$ admit a basis of Stein neighborhoods in $X$, and

(ii) $\overline{A \backslash B} \cap \overline{B \backslash A}=\emptyset$ (the separation property). 
The main step is to extend a holomorphic section (by appproximation) to a special convex bump that we now introduce (see Fig. 2). The CAP property of the fiber will be used only at this point of the proof.

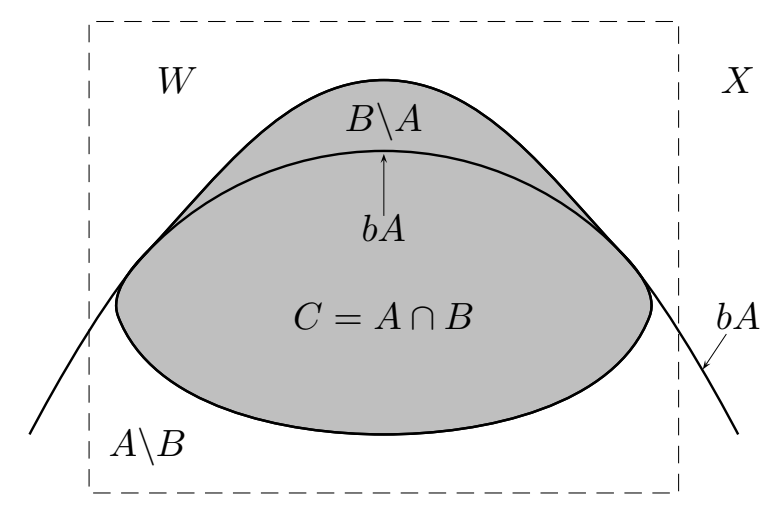

Figure 2. A special convex bump $B$ in a window $W$

Definition 5.2. Let $X$ be a complex space. A pair of compact sets $(A, B)$ in $X$ is a special Cartan pair, and $B$ is a special convex bump on $A$, if

(i) $(A, B)$ is a Cartan pair in $X$, and

(ii) there are a compact set $W \subset X_{\text {reg }}$ (a window for $B$ ), containing $B$ in its interior, and a holomorphic coordinate map $\phi$ from an open neighborhood of $W$ to $\mathbb{C}^{n}$ such that $Q=\phi(W)$ is a cube (1.1), and such that the sets

$$
K=\phi(A \cap W), \quad K^{\prime}=\phi((A \cup B) \cap W)
$$

are special convex sets in $Q$ of the form (1.2).

By subdividing $\left[s_{0}, s_{1}\right]$ into finitely many subintervals and replacing $\left[s_{0}, s_{1}\right]$ by one such subinterval we can assume that $D_{s_{1}}$ is obtained by attaching to $D_{s_{0}} \cap D_{s_{1}}$ finitely many special convex bumps contained in $X \backslash M_{1}$ (see Fig. 3). The proof is completely elementary (see e.g. [34, Lemma 12.3]).

On each bump we apply [14, Proposition 3.1] to extend the holomorphic homotopy (by approximation) across the attached bump, without changing its values on the subvariety $M_{1}$. In finitely many steps we accomplish our task.

The critical case: $h$ has a unique critical point $p \in \Omega_{s_{0}, s_{1}}$. We can extend the holomorphic homotopy across the critical level $\{h=h(p)\}$ by a reduction to 


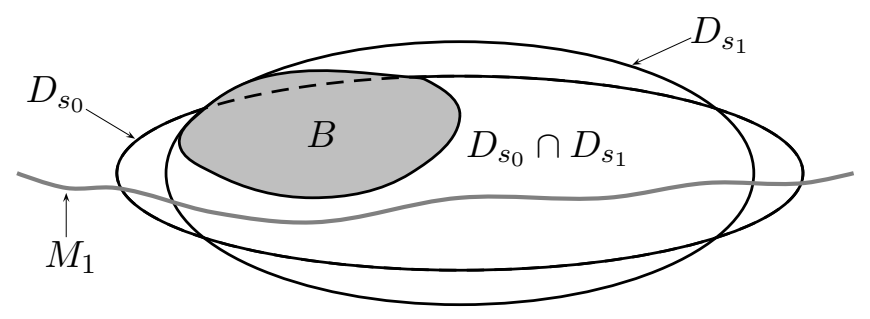

Figure 3. A special bump $B$ on $D_{s_{0}} \cap D_{s_{1}}$.

the noncritical case as in $[14$, p. 697]. This reduction was developed in $[12, \S 7.4]$ where the reader can find complete details.

Proposition 5.1 is obtained in finitely many steps of the above two types.

The general case: Now $X$ is a Stein space and $M_{1} \subset M_{0}$ are closed complex (hence Stein) subvarieties of $X$ such that $S=M_{0} \backslash M_{1}$ is a complex manifold, possibly disconnected. By the assumption we have a section $f: X \rightarrow Z$ which is holomorphic in an open set $U \subset X$ containing $K_{1}=K \cup\left(M_{1} \cap L\right)$. As before, we may assume that $L=\{x \in X: \rho(x) \leq 0\}$, where $\rho: X \rightarrow \mathbb{R}$ is a smooth strongly plurisubharmonic exhaustion function whose restriction to $S=M_{0} \backslash M_{1}$ has no critical points on $b L \cap S=\{\rho=0\} \cap S$. Note that $L$ intersects at most finitely many connected components of $S$, and hence we can embed a relatively compact neighborhood of $L$ in $X$ holomorphically into $\mathbb{C}^{N}$ (see [1]).

Since $K_{1}$ is $\mathcal{O}(X)$-convex, there is a smooth strongly plurisubharmonic function $\tau: X \rightarrow \mathbb{R}$ such that $\tau<0$ on $K_{1}$ and $\tau>0$ on $X \backslash U$. Set

$$
D_{0}=\left\{x \in M_{0}: \tau(x) \leq 0\right\} \subset U .
$$

By general position we may assume that 0 is a regular value of $\left.\tau\right|_{S}$ and the real hypersurfaces $\{\rho=0\} \cap S=b L \cap S$ and $\{\tau=0\} \cap S$ in $S$ intersect transversely along the submanifold $\Sigma=\{\rho=0\} \cap\{\tau=0\} \cap S$ of $S$.

We define $\rho_{s}$ as in (5.1) and set

$$
D_{s}=\left\{x \in M_{0}: \rho_{s}(x) \leq 0\right\}=\left\{x \in M_{0}: \tau(x) \leq s(\tau(x)-\rho(x))\right\} .
$$

As $s$ increases from 0 to $1, D_{s} \cap L$ increases from $D_{0} \cap L \subset M_{0}$ to $D_{1}=L \cap M_{0}$.

Like in the special case considered above we successively attach to the set $A_{0}=$ $K \cup\left(D_{0} \cap L\right)$ special convex bumps and handles, contained in the submanifold $S \cap L$, thereby reaching the set $K_{0}=K \cup\left(L \cap M_{0}\right)$ in finitely many steps. 
Note that the set $A_{0}$ is $\mathcal{O}(X)$-convex, and it contains a collar around the set $K_{1}=K \cup\left(L \cap M_{1}\right)$ in $L \cap M_{0}$.

We consider a typical step in the noncritical case. Assume that $(A, B)$ is a Cartan pair in $X$ such that $A$ has been obtained by attaching to $A_{0}$ finitely many special convex bumps and handles contained in $S \cap L$, and that $B \subset S \cap L$ is a special convex bump on $A \cap S$ (Def. 5.2). Note that $(A, B)$ is also a Cartan pair in the ambient space $\mathbb{C}^{N}$.

Assume inductively that we have a section $f: X \rightarrow Z$ that is holomorphic in a neighborhood of $K_{1}=K \cup\left(L \cap M_{1}\right)$ in $X$, and that is also holomorphic in a relative neighborhood of $A \cap S$ in the stratum $S$. By [14, Lemma 3.2] we 'thicken' $f$ over its domain of holomorphicity to a family of holomorphic sections $F(x, w)$ of $Z \rightarrow X$, depending holomorphically on a parameter $w=\left(w_{1}, \ldots, w_{p}\right)$ in an open neighborhood $O$ of the origin in some Euclidean space $\mathbb{C}^{p}$, such that $f=F(\cdot, 0), F(x, w)=f(x)$ for all $x \in M_{1}$ and $w \in O$, and $F$ is submersive in the $w$-variable for all base points $x$ in a neighborhood of $A \cap B$ in $S$.

Choose open neighborhoods $O_{3} \Subset O_{2} \Subset O_{1}$ of 0 in $\mathbb{C}^{p}$, with $O_{1} \Subset O$. By invoking the CAP property of the fiber of the bundle $\left.Z\right|_{S} \rightarrow S$ we can approximate $F$, uniformly over a neighborhood $V^{\prime}$ of $A \cap B$ in $S$ and uniformly with respect to $w \in O_{1}$, by a family $G$ of holomorphic sections, defined in a neighborhood of $B$ in $S$ and depending holomorphically on $w \in O_{1}$.

Assuming that $G$ approximates $F$ sufficiently closely, we find a neighborhood $V \Subset V^{\prime}$ of $A \cap B$ in $S$ and a holomorphic transition map of the form

$$
\gamma(x, w)=(x, \psi(x, w)), \quad x \in V, w \in O_{2}
$$

that is close to the identity map on $(A \cap B) \times O_{2}$ (depending on the closeness of $G$ to $F$ on $\left.V^{\prime} \times O_{1}\right)$ and such that

$$
F=G \circ \gamma \text { on } V \times O_{2} .
$$

For the details see [14, (3.2)].

We now consider a neighborhood of $L$ in $X$ as a complex subspace of $\mathbb{C}^{N}$. Choose a holomorphic retraction $\iota$ from an open neighborhood $U$ of $A \cap B$ in $\mathbb{C}^{N}$ onto an open neighborhood of $A \cap B$ in $S \subset \mathbb{C}^{N}$. (Such a retraction exists by Theorem 3.1 since $A \cap B$ has a Stein neighborhood basis and $S$ is a submanifold of $\mathbb{C}^{N}$.) We choose $U$ small enough such that $\iota(U) \subset V$. Let

$$
\widetilde{\gamma}(z, w)=\gamma(\iota(z), w), \quad z \in U, w \in O_{2} .
$$


Choose a Cartan pair $(\widetilde{A}, \widetilde{B})$ in $\mathbb{C}^{N}$ such that $A \subset \operatorname{Int} \widetilde{A}, B \subset \operatorname{Int} \widetilde{B}$, and $\widetilde{A} \cap \widetilde{B} \subset U$. (We may take $\widetilde{A}$ and $\widetilde{B}$ to be smooth strongly pseudoconvex domains; see [12, Lemma 4.3].) Assuming as we may that $\widetilde{\gamma}$ is sufficiently close to the identity map (this is insured by approximating $F$ sufficiently well by $G$ ), Lemma 2.1 in [14] furnishes a splitting

$$
\widetilde{\gamma}=\beta \circ \alpha^{-1},
$$

where $\alpha$ and $\beta$ are biholomorphic maps of the same type as $\gamma$ and close to the identity on $\widetilde{A} \times O_{3}$, resp. on $\widetilde{B} \times O_{3}$. In addition, $\alpha$ can be chosen to match the identity map to a given finite order along the intersection of its domain with the subvariety $M_{1}$. (For a simple proof of this splitting lemma see [17, Lemma 3.2].)

From $F=G \circ \gamma=G \circ \beta \circ \alpha^{-1}$ we infer that

$$
F \circ \alpha=G \circ \beta \quad \text { on }(A \cap B) \times O_{3} .
$$

This gives a family of holomorphic sections $\widetilde{F}$ of $Z \rightarrow X$ over the set $A \cup B$ such that the section $\widetilde{F}(\cdot, 0)$ approximates $f$ uniformly on $A$, it agrees with $f$ to order $\ell$ along $M_{1}$, and is homotopic to $f$ by a homotopy satisfying the required properties. The induction may now proceed.

We deal with the critical points of the function $h=\frac{\tau}{\tau-\rho}$ in $S \cap L$ exactly as before by reducing to the noncritical case (see the critical case above).

In finitely many such steps we obtain a homotopy $\left\{f_{t}\right\}_{t \in[0,1]}$ with the required properties such that $f_{1}$ is holomorphic in a neighborhood of $K_{1}$ in $X$, and also in a relative neighborhood of $L \cap M_{0}$ in the subvariety $M_{0}$.

By Proposition 4.1 there is a holomorphic section $\widetilde{f}_{1}$ in a neighborhood of $K_{0}=K \cup\left(L \cap M_{0}\right)$ in $X$ such that $\tilde{f}_{1}$ is as close as desired to $f_{1}$ on $K, \widetilde{f}_{1}=f_{1}$ on $L \cap M_{0}$, and $\widetilde{f_{1}}$ agrees with $f_{1}$ to order $\ell$ along $L \cap M_{1}$. Replace $f_{1}$ by $\widetilde{f}_{1}$ and adjust the homotopy $\left\{f_{t}\right\}$ accordingly. By using a cut-off function in the parameter of the homotopy we can extend $\left\{f_{t}\right\}$ continuously to all of $X$ without changing it near $K_{0}$ and on $M_{1}$. This completes the proof.

\section{The PARAMETRIC OKA PRINCIPle}

We have already seen in the proof of Theorem 1.4 that the basic Oka principle with interpolation also implies the 1-parametric Oka principle, the latter meaning that the inclusion

$$
\iota: \Gamma_{\mathcal{O}}(X, Z) \hookrightarrow \Gamma(X, Z)
$$


of the space of holomorphic sections into the space of continuous sections induces a bijection of the path connected components of the two spaces (Corollary 1.5).

A stronger form of the Oka principle is to demand that the inclusion (6.1) is a weak homotopy equivalence, i.e., it induces isomorphisms of all homotopy groups

$$
\pi_{k}(\iota): \pi_{k}\left(\Gamma_{\mathcal{O}}(X, Z)\right) \stackrel{\approx}{\longrightarrow} \pi_{k}(\Gamma(X, Z)), \quad k=0,1,2, \ldots
$$

To formulate the general parametric Oka principle we recall the following notions from [23, Definition 3].

Definition 6.1. Let $h: Z \rightarrow X$ be a holomorphic map of complex spaces, and let $P_{0} \subset P$ be topological spaces.

(a) A P-section of $\pi: Z \rightarrow X$ is a continuous map $f: X \times P \rightarrow Z$ such that $f_{p}=f(\cdot, p): X \rightarrow Z$ is a section of $\pi$ for each fixed $p \in P$. A $P$-section $f$ is holomorphic on $X$ (resp. on a subset $U \subset X$ ) if $f_{p}$ is holomorphic on $X$ (resp. on $U$ ) for each fixed $p \in P$.

(b) A homotopy of $P$-sections is a $P \times[0,1]$-section, i.e., a continuous map $H: X \times P \times[0,1] \rightarrow Z$ such that $H_{t}=H(\cdot, \cdot, t): X \times P \rightarrow Z$ is a $P$ section for each $t \in[0,1]$. Such homotopy $H$ is holomorphic if $H_{p, t}=$ $H(\cdot, p, t): X \rightarrow Z$ is holomorphic for each fixed $(p, t) \in P \times[0,1]$.

(c) A $\left(P, P_{0}\right)$-section of $\pi: Z \rightarrow X$ is a $P$-section $f: X \times P \rightarrow Z$ such that $f_{p}: X \rightarrow Z$ is holomorphic on $X$ for each $p \in P_{0}$. A $\left(P, P_{0}\right)$-section is holomorphic on $U \subset X$ if $\left.f_{p}\right|_{U}$ is holomorphic for every $p \in P$.

A $P$-map $X \rightarrow Y$ to a complex space $Y$ is a map $X \times P \rightarrow Y$ (which is the same thing as a $P$-section of the product fibration $Z=X \times Y \rightarrow X)$. Similarly one defines $\left(P, P_{0}\right)$-maps and their homotopies.

Given a compact subset $K$ in a complex space $X$ and a closed complex subvariety $X^{\prime}$ of $X$, we say that a $P$-section $f: X \times P \rightarrow Z$ is holomorphic on $K \cup X^{\prime}$ if there is an open neighborhood $U \subset X$ of $K$ such that for every $p \in P, f_{p}=f(\cdot, p)$ is holomorphic in $U$ and $\left.f_{p}\right|_{X^{\prime}}$ is holomorphic on $X^{\prime}$. Similar terminology applies to $\left(P, P_{0}\right)$-sections and their homotopies.

Definition 6.2. Let $\pi: Z \rightarrow X$ be a holomorphic map onto a complex space $X$, and let $d$ be a distance function on $Z$. We say that sections of $\pi$ satisfy the parametric Oka principle with approximation and interpolation (POPAI) for a given pair of topological spaces $P_{0} \subset P$ if the following holds. Given a compact $\mathcal{O}(X)$-convex set $K$ in $X$, a closed complex subvariety $X^{\prime}$ of $X$, and a 
$P$-section $f: X \times P \rightarrow Z$ that is holomorphic on $K \cap X^{\prime}$, there exists for every $\epsilon>0$ a homotopy of $P$-sections $F: X \times P \times[0,1] \rightarrow Z$ such that, setting $f^{t}=F(\cdot, \cdot, t): X \times P \rightarrow Z(t \in[0,1])$, we have

(i) $f^{0}=f$ is the initial $P$-section,

(ii) the $P$-section $f^{1}$ is holomorphic on $X$,

(iii) for each $t \in[0,1]$ the $P$-section $f^{t}$ is holomorphic on $K \cup X^{\prime},\left.f_{p}^{t}\right|_{X^{\prime}}=\left.f_{p}^{0}\right|_{X^{\prime}}$ for each $p \in P, \sup _{x \in K, p \in P} d(F(x, p, t), f(x, p))<\epsilon$, and

(iv) if $f$ is a $\left(P, P_{0}\right)$-section then $F$ can be chosen fixed on $P_{0}$ (i.e., such that $f_{p}^{t}$ is independent of $t \in[0,1]$ for each fixed $\left.p \in P_{0}\right)$.

By deleting the approximation and/or the interpolation condition in POPAI we get POP (the parametric Oka principle), POPA (the parametric Oka principle with approximation), or POPI (the parametric Oka principle with interpolation).

We also define the parametric Oka principle with approximation and jet interpolation (POPAJI) by asking that for every $P$-section $f$ which is holomorphic in an open set $U \supset K \cup X^{\prime}$ there exist holomorphic $P$-sections $\tilde{f}$ that approximate $f$ uniformly on $K$, and that agree with $f$ to a given finite order along the subvariety $X^{\prime}$. (The above terminology was introduced by Lárusson [40].)

Note that the validity of POPAJI for all finite polyhedral pairs $P_{0} \subset P$ is equivalent to Gromov's $\mathrm{Ell}_{\infty}$ property [31, 33.1$]$.

The following observation is due to Gromov [31].

Proposition 6.3. If sections of a holomorphic map $\pi: Z \rightarrow X$ satisfy the parametric Oka principle (POP) for all pairs $P_{0} \subset P$ consisting of a finite polyhedron $P$ and a subpolyhedron $P_{0}$, then the inclusion $\iota: \Gamma_{\mathcal{O}}(X, Z) \hookrightarrow \Gamma(X, Z)$ is a weak homotopy equivalence.

Proof. Taking $P=S^{k}$ (the $k$-sphere) and $P_{0}=\emptyset$, POP implies that each continuous map $S^{k} \rightarrow \Gamma(X, Z)$ can be homotopically deformed to a map $S^{k} \rightarrow \Gamma_{\mathcal{O}}(X, Z)$. Hence (6.2) is surjective.

Similarly, taking $P$ to be the closed real $(k+1)$-ball $B^{k+1}$ and $P_{0}=b B^{k+1}=S^{k}$, we conclude that each map $S^{k} \rightarrow \Gamma_{\mathcal{O}}(X, Z)$ that extends to a map $B^{k+1} \rightarrow$ $\Gamma(X, Z)$ also extends to a map $B^{k+1} \rightarrow \Gamma_{\mathcal{O}}(X, Z)$. Hence (6.2) is injective.

We now introduce a parametric version of CAP. 
Definition 6.4. A complex manifold $Y$ enjoys the Parametric Convex Approximation Property (PCAP) for a certain pair of topological spaces $P_{0} \subset P$ if for every special convex pair $(K, Q)$, a map $f: Q \times P \rightarrow Y$ such that $f_{p}=f(\cdot, p): Q \rightarrow$ $Y$ is holomorphic for every $p \in P_{0}$, and is holomorphic on $K$ for every $p \in P$, can be approximated uniformly on $K \times P$ by maps $\widetilde{f}: Q \times P \rightarrow Y$ such that $\widetilde{f}_{p}$ is holomorphic on $Q$ for all $p \in P$, and $\widetilde{f}_{p}=f_{p}$ for all $p \in P_{0}$.

The following is a parametric version of Theorem 1.4.

Theorem 6.5. If $\pi: Z \rightarrow X$ is a stratified holomorphic fiber bundle over a Stein space $X$ all of whose fibers satisfy PCAP for a certain pair of compact Hausdorff spaces $P_{0} \subset P$, then sections $X \rightarrow Z$ satisfy the parametric Oka principle with approximation and (jet) interpolation for the same pair $\left(P, P_{0}\right)$ :

$$
\text { PCAP } \Longrightarrow \text { POPAI, } \quad \text { PCAP } \Longrightarrow \text { POPAJI. }
$$

Remark 6.6. In previous papers [21, 22, 23, 10, 14, 15], POP was only considered for pairs of parameter spaces $P_{0} \subset P$ such that

$\left(^{*}\right) P$ is a nonempty compact Hausdorff space, and $P_{0}$ is a closed subset of $P$ that is a strong deformation neighborhood retract (SDNR) in $P$.

One can dispense with the SDNR condition by using a generalized Tietze extension theorem for maps into Hilbert spaces [19, Proposition 4.1]

Remark 6.7. For all compact parameter spaces $P_{0} \subset P$ contained in a Euclidean space $\mathbb{R}^{m}$ we also have the implication

$$
\mathrm{CAP} \Longrightarrow \mathrm{PCAP}
$$

(see the note at the end of the paper).

Among the conditions implying PCAP for all pairs of compact Hausdorff spaces $P_{0} \subset P$ we mention Gromov's ellipticity (the existence of a dominating holomorphic spray on $Y$, see [31]), and subellipticity (the existence of a finite dominating family of holomorphic sprays on $Y$, see [10]). Each of these conditions actually implies the parametric Oka principle for sections of any holomorphic fiber bundle $Z \rightarrow X$ with fiber $Y$ over a Stein manifold $X$ (see [10, 21, 22, 23, 31]). Like in the basis case considered above, the proof splits in two parts:

- subellipticity $\Longrightarrow$ PCAP, and

- $\mathrm{PCAP} \Longrightarrow$ POPAI. 
The first of these implications generalizes the Oka-Weil approximation theorem (see Theorem 4.1 in $[21$, p. 135] and Theorem 3.1 in [10, p. 534]). The main part of the proof is the second implication which is indeed an equivalence (for the converse implication POPAI $\Rightarrow$ PCAP it suffices to apply POPAI with $K$ a compact convex set in $X=\mathbb{C}^{n}$ and $X^{\prime}=\emptyset$ ).

Sketch of proof of Theorem 6.5. We follow the proof of Theorem 1.4, using the parametric versions of the technical ingredients at every step. There are two types of basic steps (see the proof of Proposition 5.1):

(1) extension to a special convex bump (the noncritical case);

(2) extension to a handle with a totally real core (the critical case).

Step (1) consists of three substeps:

(i) holomorphic approximation on a special convex pair,

(ii) finding a holomorphic transition map between the old and the new section,

(iii) splitting the transition map and gluing the pair of sections.

Each point $p_{0} \in P$ has an open neighborhood $U_{p_{0}} \subset P$ such that these steps can be performed simultaneously for all sections $\left\{f_{p}: p \in U_{p_{0}}\right\}$, with a continuous dependence on the parameter. Indeed, for (i) we use the hypothesis PCAP, (ii) follows from the implicit function theorem, and (iii) follows from [17, Lemma 3.2] that allows continuous dependence on parameters.

Step (2) also involves three substeps:

(a) extending the section across the core of the handle,

(b) approximation by a section that is holomorphic on a handlebody,

(c) passing the critical level by the noncritical procedure, applied with a different strongly plurisubharmonic function.

Substep (a) is automatically fulfilled since we have a global $P$-section $X \times P \rightarrow$ $Z$ at each step.

For Substep (b) we apply the parametric version of [13, Theorem 3.2] (see also [24, Theorem 4.1]).

For Substep (c) we apply Step (1) finitely many times.

We conclude the proof (globalization with respect to the parameter $p \in P$ ) by following the stepwise extension method as in the Conclusion of the proof of Theorem 4.2 in [19]. 
Corollary 6.8. If $Z \rightarrow X$ is a stratified holomorphic fiber bundle over a Stein space $X$ all of whose fibers enjoy PCAP, then the inclusion

$$
\iota: \Gamma_{\mathcal{O}}(X, Z) \hookrightarrow \Gamma(X, Z)
$$

is a weak homotopy equivalence. This holds in particular if all fibers are elliptic in the sense of Gromov [31], or subelliptic in the sense of [10].

\section{Existence of GLOBAL HOLOMORPhiC SECTIONS}

We now add a connectivity hypothesis on fibers in a stratified fiber bundle to obtain existence theorems for holomorphic sections.

Theorem 7.1. Assume that $X$ is a finite dimensional Stein space, $\pi: Z \rightarrow X$ is a holomorphic submersion, and $X=X_{0} \supset X_{1} \supset \cdots \supset X_{m}=\emptyset$ is a stratification of $X$ such that for each connected component $S$ of $X_{k} \backslash X_{k+1}$ the restriction $\left.Z\right|_{S} \rightarrow S$ is a holomorphic fiber bundle whose fiber $Y_{S}$ enjoys CAP and $\pi_{q}\left(Y_{S}\right)=0$ for $q \in\{1,2, \ldots, \operatorname{dim} S-1\}$. Then there exists a holomorphic section $X \rightarrow Z$.

Furthermore, given a closed complex subvariety $X^{\prime} \subset X$, a compact $\mathcal{O}(X)$ convex subset $K \subset X$, an open set $U \supset K$, and a holomorphic section $f: U \cup X^{\prime} \rightarrow$ $Z$, there exists a holomorphic section $\widetilde{f}: X \rightarrow Z$ with $\left.\widetilde{f}\right|_{X^{\prime}}=\left.f\right|_{X^{\prime}}$ such that $\tilde{f}$ approximates $f$ as close as desired uniformly on $K$.

Proof. The only place in the proof of Theorem 1.4 that requires a topological condition on the fiber is when crossing a critical point $p$ of index $k \geq 1$ of a strongly plurisubharmonic Morse function $\rho$ on a stratum $S$ (see the critical case in the proof of Proposition 5.1).

To cross the critical level of $\rho$ at $p$ we must be able to extend a given section, defined on a sublevel set $\{\rho \leq c\}$ for some $c<\rho(p)$ close to the critical level $\rho(p)$, to a continuous section over a $k$-dimensional totally real disc $E$ in $S$, attached with its boundary sphere $b E \approx S^{k-1}$ to the hypersurface $\{\rho=c\}$, such that $\{\rho \leq c\} \cup E$ is a strong deformation retraction of a sublevel set $\left\{\rho \leq c^{\prime}\right\}$ for some $c^{\prime}>\rho(p)$. Such an extension exists if and only if the map $f: b E \rightarrow Y$ is null-homotopic in $Y$, and this is certainly the case if the group $\pi_{k-1}(Y)$ vanishes.

Since $\left.\rho\right|_{S}$ is strongly plurisubharmonic, we have $k \leq \operatorname{dim} S$. Our topological assumption on the fibers of $Z \rightarrow X$ therefore insures the existence of a continuous extension of a section at each critical point on every stratum, and hence the proof of Theorem 1.4 applies. 
Corollary 7.2. Assume that $X$ is a Stein manifold of dimension $n, K$ is a compact $\mathcal{O}(X)$-convex subset of $X, X^{\prime}$ is a closed complex subvariety of $X, U$ is an open set in $X$ containing $K$, and $f: U \cup X^{\prime} \rightarrow Y$ is a holomorphic map. If $Y$ enjoys $\mathrm{CAP}$ and if $\pi_{q}(Y)=0$ for $q=0,1, \ldots, n-1$, then there exists a holomorphic map $\tilde{f}: X \rightarrow Y$ such that $\left.\widetilde{f}\right|_{X^{\prime}}=\left.f\right|_{X^{\prime}}$ and $\tilde{f}$ approximates $f$ uniformly on $K$ as close as desired. The conclusion holds if $Y=\mathbb{C}^{N} \backslash A$, where $A$ is a closed algebraic subvariety of $\mathbb{C}^{N}$ of codimension $q=N-\operatorname{dim} A \geq \max \left\{2, \frac{n+1}{2}\right\}$.

Proof. The first conclusion is a special case of Theorem 7.1. For the second part, recall that $\mathbb{C}^{N} \backslash A$ enjoys CAP if $q \geq 2$ (see [14, Corollary 1.3]), and $\pi_{k}\left(\mathbb{C}^{N} \backslash A\right)=0$ when $0 \leq k \leq 2 q-2$ (this follows from a general position argument).

\section{SubMERSIONS WITH STRATIFIED SPRAYS}

Assume that $\pi: Z \rightarrow X$ is a holomorphic submersion of finite dimensional complex spaces (Def. 1.2). For any point $x \in X$ we set $Z_{x}=\pi^{-1}(x)$. For $z \in Z_{x}$ we denote by $V T_{z} Z=T_{z} Z_{x}$ the tangent space to the fiber $Z_{x}$, also called the vertical tangent space of $Z$ at $z$ (with respect to $\pi$ ).

Definition 8.1. (Gromov [31]) Assume that $\pi: Z \rightarrow X$ is a holomorphic submersion of complex spaces. A fiber dominating spray associated to this submersion is a triple $(E, p, s)$, where $p: E \rightarrow Z$ is a holomorphic fiber bundle and $s: E \rightarrow Z$ is a holomorphic map satisfying the following properties for every $z \in Z$ :

(i) $s\left(0_{z}\right)=z$ (here $0_{z} \in E_{z}$ is the zero element of the fiber of $E$ over $z$ ),

(ii) $\pi \circ s=\pi \circ p$ (i.e., $\left.s\left(E_{z}\right) \subset Z_{\pi(z)}\right)$, and

(iii) $(d s)_{0_{z}}\left(E_{z}\right)=V T_{z} Z$.

Condition (iii), which means that the map $s: E_{z} \rightarrow Z_{\pi(z)}$ is a submersion at $0_{z} \in E_{z}$, is called the domination property of the spray.

Definition 8.2. A holomorphic submersion $\pi: Z \rightarrow X$ admits stratified sprays (or is a stratified elliptic submersion) if there exists a stratification $X=X_{0} \supset$ $X_{1} \supset \cdots \supset X_{m}=\emptyset$ such that each point $x$ in any stratum $S \subset X_{k} \backslash X_{k+1}$ has an open neighborhood $U$ in $S$ such that the restricted submersion $\pi:\left.Z\right|_{U} \rightarrow U$ admits a fiber dominating spray (Def. 8.1).

A special case of the following result was given in $[23$, p. 66]. 
Theorem 8.3. Let $\pi: Z \rightarrow X$ be a holomorphic submersion of finite dimensional complex spaces. If $X$ is Stein and if the submersion admits stratified sprays (Def. 8.2), then sections $X \rightarrow Z$ satisfy the parametric Oka principle with approximation and (jet) interpolation for any pair of compact Hausdorff spaces $P_{0} \subset P$ (see Def. 6.2). The conclusion of Corollary 6.8 holds as well.

The validity of Theorem 8.3 was indicated by Gromov [31]. In [22, 23] a proof was given for the case when the base $X$ is a Stein manifold and there is only one stratum. The methods of this paper allow us to complete the proof also for Stein spaces with singularities, and for submersions with stratified sprays, along the lines indicated in $[23, \S 7]$.

Proof. We follow the proof of Theorem 1.4 (see $\S 5$ above). Lets us consider the basic case without parameteres. At a typical step of the induction we have a section $f: X \rightarrow Z$ that is holomorphic on a neighborhood of a compact $\mathcal{O}(X)$ convex subset $K$ of $X$, and whose restriction to a closed complex subvariety $X^{\prime}$ of $X$ is holomorphic on $X^{\prime}$. Given a larger compact $\mathcal{O}(X)$-convex subset $L$ in $X$ containing $K$, our task is to homotopically deform $f$ to a section that is holomorphic in a neighborhood of $L$ such that the homotopy is fixed on $X^{\prime}$ and such that all sections in the homotopy are holomorphic near $K$ and are uniformly close to $f$ on $K$. The proof is then completed by induction over a sequence of compacta exhausting $X$.

Applying Proposition 4.1 we first modify $f$ to make it holomorphic in an open neighborhood of $K \cup X^{\prime}$. Let $X=X_{0} \supset X_{1} \supset \cdots \supset X_{m}=\emptyset$ be a stratification satisfying the hypotheses of the theorem. Replacing $X_{k}$ by $X_{k}^{\prime}=X_{k} \cup X^{\prime}$ we obtain another stratification

$$
X=X_{0}^{\prime} \supset X_{1}^{\prime} \supset \cdots \supset X_{m}^{\prime}=X^{\prime}
$$

with regular strata $X_{k}^{\prime} \backslash X_{k+1}^{\prime}=X_{k} \backslash\left(X_{k+1} \cup X^{\prime}\right) \subset X_{k} \backslash X_{k+1}$ such that the dominating spray condition still holds over the strata.

Assume inductively that $f$ is already holomorphic in a neighborhood of $K \cup$ $\left(X_{k+1}^{\prime} \cap L\right)$ (this holds when $k+1=m$ ). The goal is to modify $f$ to a section that is holomorphic in a neighborhood of $K \cup\left(X_{k}^{\prime} \cap L\right)$ such that the deformation is fixed on $X_{k+1}^{\prime}$ and is small on $K$; the proof is then completed by a finite downward induction on $k$. This is accomplished by Proposition 6.1 in [23] as follows. 
We choose a Cartan string $\left(A_{0}, A_{1}, \ldots, A_{n}\right)$ in the subvariety $X_{k}^{\prime}$ such that $A_{0}$ is a (small) neighborhood of $K \cup\left(X_{k+1}^{\prime} \cap L\right)$ on which $f$ is holomorphic, while the remaining sets $A_{1}, \ldots, A_{n}$ are contained in the strata $S=X_{k}^{\prime} \backslash X_{k+1}^{\prime}$ (hence in the regular locus of $X_{k}^{\prime}$ ), and they are chosen sufficiently small such that the restricted submersion $\left.Z\right|_{S} \rightarrow S$ admits a fiber dominating spray on a neighborhood of each of them. In addition, we require that $\bigcup_{j=0}^{n} A_{j}=L \cap X_{k}^{\prime}$. (For the definition and the construction of such Cartan strings see Gromov [31, 4.2.D'] and [22], Def. 4.1 and Theorem 4.6.) Now we obtain a desired modification of $f$ by applying Proposition 5.1 in [22] with the given Cartan string.

The proof in the parametric case follows the same lines (see $\S 6$ above and $[22,23])$.

Remark 8.4. Two further improvements of Theorem 8.3 are possible.

As in Theorem 1.4, it suffices that the assumptions of Theorem 8.3 hold over an exhausting sequence of open relatively compact subsets of $X$. This allows $X$ to be infinite dimensional.

Secondly, the fiber domination condition can be weakened by asking that each point $x$ in a stratum $S$ (in a given stratification) has an open neighborhood $U \subset S$ such that the restricted submersion $\left.Z\right|_{U} \rightarrow U$ admits a finite collection of holomorphic sprays $\left(E_{j}, p_{j}, s_{j}\right), j=1, \ldots, k$, satifying the following domination condition at every point $\left.z \in Z\right|_{U}=\pi^{-1}(U)$ :

$$
\left(d s_{1}\right)_{0_{z}}\left(E_{1, z}\right)+\left(d s_{2}\right)_{0_{z}}\left(E_{2, z}\right)+\ldots+\left(d s_{k}\right)_{0_{z}}\left(E_{k, z}\right)=V T_{z} Z
$$

A submersion $Z \rightarrow X$ satisfying this property is called a stratified subelliptic submersion. We refer to [10] for further details.

Acknowledgement. I wish to thank Jasna Prezelj for stimulating discussions on this subject over many years, and Frank Kutzschbauch for telling me about the solution to the holomorphic Vaserstein problem which prompted me to include $\S 8$ to the paper. Finally, I thank the referee for his helful remarks that lead me to include a discussion of the parametric case.

Added to the page proofs. Recently I showed in [18] that the convex approximation property CAP (see Def. 1.1) implies the parametric convex approximation property PCAP (see Def. 6.4) for all pairs of compact parameter sets $P_{0} \subset P$ contained in some Euclidean space $\mathbb{R}^{m}$. Such pairs suffice for all known applications. By abstract homotopy theory (see [39]) one also gets from this PCAP for 
all pairs $\left(P, P_{0}\right)$ consisting of a finite $\mathrm{CW}$-complex $P$ and a subcomplex $P_{0}$. For such pairs $P_{0} \subset P$, Theorem 6.5 holds under the assumption that all its fibers satisfy CAP.

\section{REFERENCES}

1. E. BISHOP, Mappings of partially analytic spaces. Amer. J. Math. 83 (1961), 209-242.

2. H. CARTAN, Espaces fibrés analytiques. In: Symposium Internat. de topologia algebraica (Mexico, 1958), 97-121. Also in: Cartan, H.: Oeuvres, 2, Springer-Verlag, New York, 1979.

3. M. COLŢOIU, Complete locally pluripolar sets. J. Reine Angew. Math., 412 (1990), 108112.

4. M. COLŢOIU, N. MIHALACHE, On the homology groups of Stein spaces and Runge pairs. J. Reine Angew. Math., 371 (1986), 216-220.

5. J.-P. DEMAILLY, Cohomology of $q$-convex spaces in top degrees. Math. Z., 204 (1990), 283-295.

6. F. DOCQUIER, H. GRAUERT, Levisches Problem und Rungescher Satz für Teilgebiete Steinscher Mannigfaltigkeiten. Math. Ann., 140 (1960), 94-123.

7. Y. ELIASHBERG, M. GROMOV: Embeddings of Stein manifolds. Ann. Math. (2) 136 (1992), 123-135.

8. O. FORSTER, K. J. RAMSPOTT, Okasche Paare von Garben nichtabelscher Gruppen. Invent. Math., 1 (1966), 260-286.

9. O. FORSTER, K. J. RAMSPOTT, Analytische Modulgarben und Endromisbündel. Invent. Math., 2 (1966), 145-170.

10. F. FORSTNERIČ, The Oka principle for sections of subelliptic submersions. Math. Z., 241 (2002), 527-551.

11. F. FORSTNERIČ, The Oka principle for multivalued sections of ramified mappings. Forum Math., 15 (2003), 309-328.

12. F. FORSTNERIČ, Noncritical holomorphic functions on Stein manifolds. Acta Math., 191 (2003), 143-189.

13. F. FORSTNERIČ, Holomorphic submersions from Stein manifolds. Ann. Inst. Fourier, 54 (2004), 1913-1942.

14. F. FORSTNERIČ, Runge approximation on convex sets implies Oka's property. Ann. Math., (2) 163 (2006), 689-707.

15. F. FORSTNERIČ, Extending holomorphic mappings from subvarieties in Stein manifolds. Ann. Inst. Fourier, 55 (2005), 733-751.

16. F. FORSTNERIČ, Holomorphic flexibility properties of complex manifolds. Amer. J. Math., 128 (2006), 239-270.

17. F. FORSTNERIČ, Manifolds of holomorphic mappings from strongly pseudoconvex domains. Asian J. Math., 11 (2007), No. 1, 113-126.

18. F. FORSTNERIČ, Oka Manifolds. C. R. Acad. Sci. Paris, Ser. I 347 (2009), 1017-1020. 
19. F. FORSTNERIČ, Invariance of the parametric Oka property. (Proc. of the conference in honor of Linda P. Rothschild, Fribourg, Switzerland, July 2008), Birkhäuser Verlag, to appear. arXiv: Math.CV 0705.0591.

20. F. FORSTNERIČ, B. IVARSSON, F. KUTZSCHEBAUCH, J. PREZELJ, An interpolation theorem for proper holomorphic embeddings. Math. Ann., 338 (2007), 545-554.

21. F. FORSTNERIČ, J. PREZELJ, Oka's principle for holomorphic fiber bundles with sprays. Math. Ann., 317 (2000), 117-154.

22. F. FORSTNERIČ, J. PREZELJ, Oka's principle for holomorphic submersions with sprays. Math. Ann., 322 (2002), 633-666.

23. F. FORSTNERIČ, J. PREZELJ, Extending holomorphic sections from complex subvarieties. Math. Z., 236 (2001), 43-68.

24. F. FORSTNERIČ, M. SLAPAR, Stein structures and holomorphic mappings. Math. Z., 256 (2007), 615-646.

25. F. FORSTNERIČ, M. SLAPAR, Deformations of Stein structures and extensions of holomorphic mappings. Math. Res. Lett., 14 (2007), 343-357.

26. H. GRAUERT, Holomorphe Funktionen mit Werten in komplexen Lieschen Gruppen. Math. Ann., 133 (1957), 450-472.

27. H. GRAUERT, Approximationssätze für holomorphe Funktionen mit Werten in komplexen Räumen. Math. Ann., 133 (1957), 139-159.

28. H. GRAUERT, Analytische Faserungen über holomorph-vollständigen Räumen. Math. Ann., 135 (1958), 263-273.

29. H. GRAUERT, H. KERNER, Approximation von holomorphen Schnittflächen in Faserbündeln mit homogener Faser. Arch. Math., 14 (1963), 328-333.

30. H. GRAUERT, R. REMMERT, Theory of Stein Spaces. Grundlehren Math. Wiss. 227, Springer-Verlag, New York, 1977.

31. M. GROMOV, Oka's principle for holomorphic sections of elliptic bundles. J. Amer. Math. Soc., 2 (1989), 851-897.

32. R. C. GUNNING, H. ROSSI, Analytic functions of several complex variables. Prentice-Hall, Englewood Cliffs, 1965.

33. P. HEINZNER, F. KUTZSCHEBAUCH, An equivariant version of Grauert's Oka principle. Invent. Math., 119 (1995), 317-346.

34. G. M. HENKIN, J. LEITERER, Andreotti-Grauert theory by integral formulas. Birkhäuser, Boston, 1988 .

35. G. M. HENKIN, J. LEITERER, The Oka-Grauert principle without induction over the basis dimension. Math. Ann., 311 (1998), 71-93.

36. L. HÖRMANDER, An Introduction to Complex Analysis in Several Variables. Third ed. North Holland, Amsterdam, 1990.

37. B. IVARSSON, F. KUTZSCHEBAUCH, A solution of Gromov's Vaserstein problem. C. R. Acad. Sci. Paris, Ser. I, 336 (2008)

38. F. LÁRUSSON, Excision for simplicial sheaves on the Stein site and Gromov's Oka principle. Internat. J. Math., 14 (2003), 191-209. 
39. F. LÁRUSSON, Model structures and the Oka principle. J. Pure Appl. Algebra, 192 (2004), 203-223.

40. F. LÁRUSSON, Mapping cylinders and the Oka principle. Indiana Univ. Math. J., 54 (2005), $1145-1159$.

41. J. LEITERER, V. VÂJÂITU, A relative Oka-Grauert principle on 1-convex spaces. J. reine angew. Math., 564 (2003), 85-104.

42. K. OKA, Sur les fonctions des plusieurs variables. III: Deuxième problème de Cousin. J. Sc. Hiroshima Univ., 9 (1939), 7-19.

43. J. PREZELJ, Interpolation of embeddings of Stein manifolds on discrete sets. Math. Ann. 326 (2003), 275-296.

44. J. PREZELJ, Weakly regular embeddings of Stein spaces with isolated singularities. Pacific J. Math., 220 (2005), 141-152.

45. J. PREZELJ, A relative Oka-Grauert principle for holomorphic submersions over 1-convex spaces. Trans. Amer. Math. Soc., to appear.

46. J. SCHÜRMANN, Embeddings of Stein spaces into affine spaces of minimal dimension. Math. Ann. 307 (1997), 381-399.

47. Y.-T. SIU, Every Stein subvariety admits a Stein neighborhood. Invent. Math., 38 (1976), 89-100.

Franc Forstnerič

Faculty of Mathematics and Physics

University of Ljubljana, and Institute of Mathematics,

Physics and Mechanics, Jadranska 19, 1000 Ljubljana, Slovenia

E-mail: franc.forstneric@fmf.uni-lj.si 